\title{
Ferromagnetic/DMS hybrid structures: one- and zero-dimensional magnetic traps for quasiparticles
}

\author{
P. Redlińskif T. G. Rappoport, A. Libal, J. K. Furdyna, and B. Jankó \\ Department of Physics, University of Notre Dame, Notre Dame, IN 46556 \\ T. Wojtowicz \\ Department of Physics, University of Notre Dame, Notre Dame, IN 46556 and \\ Institute of Physics, Polish Academy of Sciences, Warsaw, Poland
}

\begin{abstract}
We investigated possibility of using local magnetic field originating from ferromagnetic island deposited on the top of semiconductor quantum well to produce zero- and one-dimensional traps for quasi-particles with spin. In particular we considered two shapes of experimentally made magnets - cylindrical and rectangular. In the case of ferromagnetic micro-disk the trap can localize spin in three dimensions, contrary to the rectangular micro-magnet which creates a trap that allows free propagation in one direction. We present in detail prediction for absorption spectrum around the main absorption edge in both type of micro-magnets.
\end{abstract}

Recently there is an increasing interest in using the spin of particles, instead of their charge, as a basis for the operation of a new type of electronic devices. In this work we show via theoretical calculations that spin degrees of freedom can be utilized for achieving spatial localization of both charged quasi-particles (electrons, holes, trions) as well as of neutral complexes (excitons). Such localized states are of interest from spintronic application point of view.

The hybrid structure we consider is build of CdMnTe/CdMgTe quantum well (QW) buried at nanometers distances $(d)$ below two types of experimentally important magnetic Fe islands: with rectangular $\underline{1}$ and cylindrical ${ }^{2}$ shape. In order to make localization effects sizeable we used diluted magnetic CdMnTe semiconductor (DMS) QW instead of classical semiconductor because of a giant Zeeman effect that exist in DMS (for a review see ref $\underline{3}$ ). Theoretically, DMS can be described

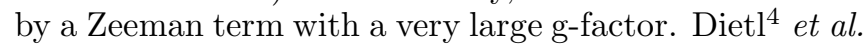
reported electron g-factor $\left|g_{e}\right|=500$ in sub-Kelvin experiment, which assuming known ratio of exchange constant in CdMnTe: $\beta / \alpha=4$, gives hole g-factor $\left|g_{h}\right|=2000$. These giant, but realistic values of g-factors were used in our calculations.

The magnetic field produced by the magnetic islands was calculated by solving magneto-static equations with magnetization distribution determined from micromagnetic simulations in the case of micro-disk, and by direct integration of Maxwell's magneto-static equations in the case of the rectangular magnet, where homogenous magnetization was assumed. Then, the energy spectrum of conduction band as well as the spectrum of a valence band were calculated by approximating the Schrödinger differential eigen-equation with a finite difference algebraic equation. In the case of $\mu$-disk, valence band electrons were modeled by Luttinger Hamiltonian. Inclusion of mixing between heavy and light holes is important because of anisotropic spin-splitting ${ }^{-5}$ of valence energy levels in DMS QW in the presence of an external magnetic field with an arbitrary direction. Using Fermi's Golden

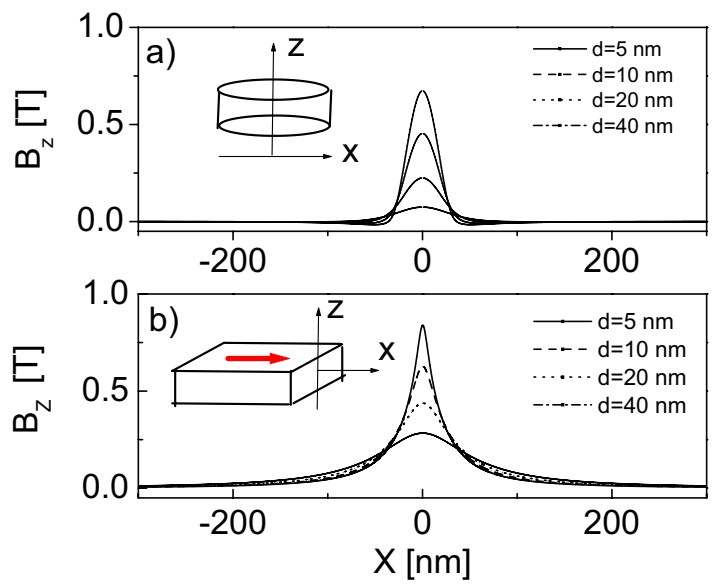

FIG. 1: Upper panel: magnetic field $B_{z}$ at a distance $d$ below the center of magnetic micro-disk. Lower panel: $B_{z}$ at distance $d$ below one of the two ends of the rectangular micromagnet. Both magnets have the same thickness $D_{z}=5 \mathrm{~nm}$. With increasing $d$ maximum of $B_{z}$ is decreasing.

Rule, together with previously calculated energies and wave functions, we could calculate absorption coefficient $\alpha(E)$. Each transition line was broadened with a Gaussian function in order to simulate the realistic absorption data.

We first consider a Fe micro-disk ${ }^{2}$ (diameter $R=1 \mu \mathrm{m}$ and thickness $\left.D_{z}=50 \mathrm{~nm}, \mu_{0} M_{s}=2.2 \mathrm{~T}\right)$ in the so called vortex state. In this state the magnetization lies mainly in plane of the disk but in its center it is forced out of plane. The diameter $R_{c}$ of the core, where magnetization is pointing out of plane, extends only over $60 \mathrm{~nm}$. It is important to mention that only $M_{z}$, the z-component of the total magnetization $\vec{M}$, produces magnetic field $\vec{B}$ which couples to the quasi-particle's spin. The calculated profiles of the $B_{z}$ component, that gives largest confinement effect, are presented in Fig. $₫$ a. At a distance of $d=10 \mathrm{~nm}$ below the Fe magnet, the maximum 
magnetic field is $|B|_{\max }=0.46 \mathrm{~T}$ and its spatial extension is over a distance $80 \mathrm{~nm}$. In Fig. „ we present $\alpha(E)$ for three distances $d$ between micro-magnetic disk and QW: $d=15 \mathrm{~nm}, d=10 \mathrm{~nm}$, and $d=5 \mathrm{~nm}$. Energy is measured relative to the energy of the main absorption edge in QW without deposition of magnetic island. Vertical bar height represent oscillator strengths of the optical transitions. As expected, low-energy peaks appear at lower energies with decreasing $d$ because the magnitude of $\vec{B}$ increases. Separation between peaks is approximately the same $(3 \mathrm{meV})$ for all three distances $d$.

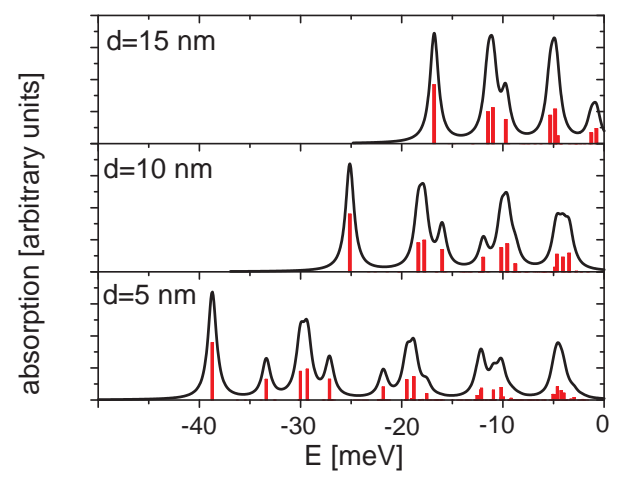

FIG. 2: Absorption spectra (full lines) of a hybrid structures composed of a Fe magnetic island deposited on the surface of DMS QW structure at distance $d=15 \mathrm{~nm}, d=10 \mathrm{~nm}$, and $d=5 \mathrm{~nm}$ from QW. In our calculations each transition line (bar) was broaden by Gaussian function with line width of 1 meV.

Next, we analyze rectangular $\mu$-magnet $\frac{1}{1}$ (size: $D_{x}=6 \mu \mathrm{m}, D_{y}=2 \mu \mathrm{m}$, and $D_{z}=0.15 \mu \mathrm{m}, \mu_{0} M_{s}=2.2 \mathrm{~T}$ ) in a single domain state, with magnetization pointing in $\mathrm{x}$-direction. Please note that the thickness of this magnet is 3 times larger than cylindrical $\mu$-disk discussed above. The corresponding field profile is plotted in Fig. $₫$ b. The magnetic field is constant along the $y$ edge of the magnet and therefore, the field produces one dimensional confining potential. In Fig. . we present the absorption spectra for three distances: $d=10 \mathrm{~nm}, d=30 \mathrm{~nm}$, and $d=60 \mathrm{~nm}$. In panel for $d=10 \mathrm{~nm}$, the numbers $(\mathrm{nm})$ indicate that corresponding line is associated with transition from $\mathrm{m}$ hole state to n-electron state. The interesting property of field-induced confining potential is that non-diagonal transitions $(n \neq m)$ are very strong, and can even be stronger than diagonal ones $(n=m)$. For example, transition (24) is stronger than transition (33), which is not even visible in this scale.

Additionally, we have performed calculations for various magnet thickness. We found that although the spatial extent of the field increases for thicker micromagnet the effect of increasing amplitude of the field is dominating and therefore the energetic distance between (11) transition and main absorption peak of $\mathrm{QW}$, as well as

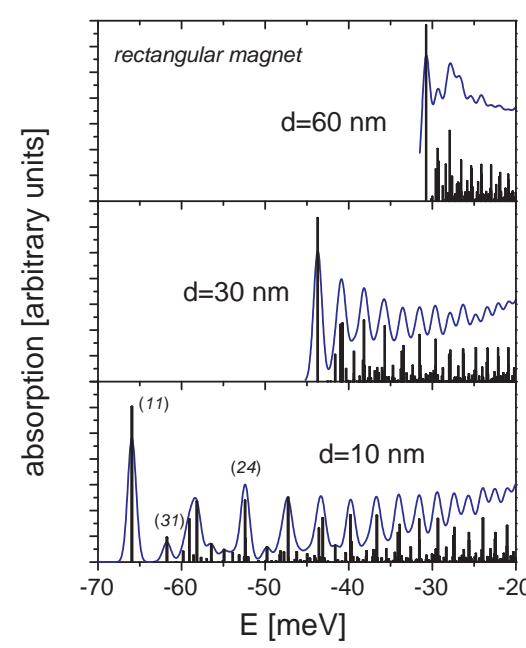

FIG. 3: Absorption spectra (full lines) in the case of rectangular ferromagnetic island at three distances $d$ between island and QW. For $d=10 \mathrm{~nm}$, numbers $(\mathrm{nm})$ indicate that given line corresponds to the transition from m-hole state to n-electron state.

distance between peaks in the spectrum increase with the increasing micromagnet thickness. Therefore it is more promising to make hybrid structures with relatively thick micromagnets.

This work was supported by the NSF-NIRT grant DMR02-01519.
* Electronic address: Pawel.Redlinski.1@nd.edu

${ }^{1}$ Kossut, J., et al., App. Phys. Lett., 79, 1789 (2001).

2 Berciu, M., and Jankó, B., Phys. Rev. Lett., 90, 246804 (2003).

3 Furdyna, J. K., and Kossut, J., Diluted Magnetic Semicon- ductor, Academic, San Diego, 1988.

4 Dietl, T., et al., Phys. Rev. B, 43, 3154 (1991).

5 Kuhn-Heinrich, B., et al., Solid State Commun., 91, 413 (1994). 